\title{
Superando fronteiras da educação com ecossistemas de aprendizagem
}

\author{
Pedro David Netto Silveira ${ }^{1}$, Davidson Cury $^{1}$, Crediné Silva de Menezes ${ }^{1}$ \\ ${ }^{1}$ Departamento de Informática \\ Universidade Federal do Espírito Santo (UFES) - Vitória, ES - Brasil \\ \{pedro.dns, dedecury, credine\}@gmail.com
}

\begin{abstract}
We are moving towards an increasingly technological education, as digital culture grows. With lot of technology and easeness of interaction, ideas arise but are lost through lack of registration, help and encouragement. The traditional school, where only part of the learning happens, still has limitations that aggravate this problem. They are the boundaries of education. In this paper, we bring as result a discussion, under the light of an ontology developed with SABiO methodology, of how learning ecosystems can help to supplant, or at least attenuate, the boundaries of education.
\end{abstract}

Resumo. Caminhamos rumo a uma educação cada vez mais tecnológica, no passo do crescimento da cultura digital. Com tanta oferta de tecnologia e facilidade de interações, as ideias surgem mas acabam se perdendo por ausência de registro, auxílio e incentivo. A escola tradicional, onde só parte da aprendizagem acontece, ainda tem limitações que agravam esse problema. São as fronteiras da educação. Neste artigo, trazemos como resultado uma discussão, sob a luz de uma ontologia desenvolvida com a metodologia SABiO, sobre como os Ecossistemas de Aprendizagem podem ajudar a superar, ou pelo menos atenuar, as fronteiras da educação.

\section{Introdução}

A tecnologia está tão presente na vida das pessoas que estabeleceu-se a chamada cultura digital [Castells 2008]. A partir da imersão de nossa sociedade na cultura digital, existe a tendência de que caminhamos rumo a uma escola digital, um movimento no qual seus integrantes poderão aprender e auxiliar a aprendizagem de outros, dentro e fora da escola, apoiados pela computação [Silveira et al. 2018b].

Grupos se formam, muitas vezes involuntariamente, para diversos fins, como: comunicação, troca de experiências, solução de problemas etc. Esses indivíduos que interagem dentro de um determinado ambiente, na maioria das vezes sequer sabem que estão em um ecossistema de aprendizagem. Com tanta oferta de tecnologia e facilidade de interações, as ideias surgem mas acabam se perdendo por ausência de registro, auxílio e incentivo. A escola tradicional tem limitações que a impossibilitam de resolver esse problema. Chamamos essas limitações de fronteiras da educação [Silveira et al. 2018a].

Concordamos com as teorias largamente exploradas pela literatura de que as aprendizagens estão intimamente ligadas às interações [Piaget et al. 1970], sejam elas entre humanos ou entre humano e os ambientes onde esteja inserido, o que nos remete à ideia de ecossistemas [ECOSSISTEMA 2019]. 
Apresentamos neste artigo uma arquitetura conceitual pertencente a uma ontologia a partir da qual podemos especializar submodelos para formalizar ecossistemas de aprendizagem. A partir destes submodelos podem ser gerados produtos a fim de potencializar as aprendizagens dos indivíduos dentro do ecossistema. Poderemos ajudar os aprendizes na coleta e registro do conhecimento produzido em diferentes lugares, na recomendação de leituras e novos parceiros, visando a suplantação das mencionadas limitações do sistema educacional atual, na busca de uma aprendizagem sem fronteiras [Silveira et al. 2018a].

Este trabalho está organizado da seguinte forma: a Seção 2 contém a fundamentação teórica; na 3 discorremos sobre os problemas enfrentados na educação sob o ponto de vista de cinco fronteiras; na 4 apresentamos uma parte de uma ontologia para ecossistemas de aprendizagem na cultura digital; na 5 discutimos como os ecossistemas de aprendizagem podem auxiliar na suplantação das fronteiras e finalmente, na Seção 6 apresentamos as considerações finais e trabalhos futuros.

\section{Fundamentação teórica}

Nesta seção discutiremos alguns conceitos necessários para o entendimento adequado do que estamos propondo, destacando-se ecossistemas de aprendizagem, cultura digital, teorias epistemológicas de Piaget e ontologias.

\subsection{Ecossistemas de aprendizagem na cultura digital}

Por definição "ecossistema"significa "um sistema formado por um meio natural (ambiente) e por uma comunidade de organismos, assim como as inter-relações de ambos" [ECOSSISTEMA 2019]. Em outras palavras, Ecossistema é a união de indivíduos com um ambiente onde ocorrem diferentes interações.

A qualidade ecológica (ou ecossistêmica) tem sido aplicada a muitos contextos e está bem adaptada às condições humanas. Nos ecossistemas humanos, a perspectiva ecológica vê as pessoas em seus ambientes físicos, sociais e virtuais, como um sistema unitário que vive dentro de um contexto histórico e cultural particular, consumindo, reciclando e produzindo recursos, incluindo informações e conhecimento, aprendendo e se desenvolvendo por meio de processos interacionais [Germain and Gitterman 1995].

A tecnologia disponível propõe facilidades de interações, na alta velocidade das redes informacionais e nas práticas de simulação. A cultura digital é uma radical mudança de era e incorpora as seguintes características: (i) muitas modalidades de comunicação global ou local em tempo real, (ii) grande aporte para realização de interações, (iii) facilidade na recuperação da informação e (iv) constituição gradual da mente coletiva pelo trabalho em rede [Castells 2008].

Definimos ecossistema de aprendizagem na cultura digital (ou simplesmente ecossistemas de aprendizagem) como a união agente/ambiente na qual acontece cognição e resultantes aprendizagens, a partir da interação de agentes (humanos e sintéticos) entre si e/ou com o ambiente. A partir de uma perspectiva ecológica, a Internet pode ser vista como parte do ecossistema para a cognição humana. Ela provê a base imaterial de suporte para o ambiente material onde atividades cognitivas e epistêmicas acontecem e se desdobram em escala planetária [Smart et al. 2017]. 
VIII Congresso Brasileiro de Informática na Educação (CBIE 2019)

Anais do XXX Simpósio Brasileiro de Informática na Educação (SBIE 2019)

\subsection{Interações e as aprendizagens}

As formas de interação entre indivíduos possibilitam trocar pontos de vista, conhecer e refletir sobre diferentes questionamentos, e então ampliar com autonomia uma tomada de consciência para buscar novos rumos [Silveira et al. 2018a].

O desenvolvimento do pensamento requer um elemento social de cooperação. Partindo desse pressuposto, [Piaget 1973] define a cooperação como "operar em conjunto". Ela é caracterizada pela coordenação de diferentes pontos de vista, pela reciprocidade e pela existência de regras de condutas que têm por princípio o respeito bilateral.

[Perret-Clermont 1980], baseado no trabalho de Piaget, concentrou-se na influência das interações sociais para o desenvolvimento cognitivo, com a suposição que a aprendizagem acontece no interior de cada um, mas é dependente das trocas sociais, e atribui às interações um papel preponderante no desenvolvimento cognitivo do sujeito. As interações são importantes no sentido que o conhecimento é construído conjuntamente, porque se produz interatividade entre duas ou mais pessoas que participam dela. Esta interação social ou interpessoal é identificada como aspecto fundamental tanto pela epistemologia genética quanto pela escola sócio-histórica [Echeita and Martin 1995].

\subsection{Ontologias}

Como apontado em [Guarino 1998], ontologias nem sempre podem ser ideais e, portanto, uma definição geral para uma ontologia (não ideal) deve ser dada: Uma ontologia é uma especificação conceitual que descreve o conhecimento sobre um domínio de uma maneira que é independente de estados epistêmicos e estado de coisas. Além disso, ela pretende restringir as possíveis interpretações do vocabulário de uma linguagem, de modo que seus modelos lógicos se aproximem tanto quanto possível do conjunto de estruturas universais pretendidas de uma conceituação desse domínio [Guizzardi 2007].

Ontologias podem ser usadas para vários fins, incluindo apoiar a especificação e implementação de qualquer sistema de computação complexo. De uma forma geral, busca-se atingir os seguintes propósitos com uma ontologia: (i) Facilitar as pessoas a transmitir seu entendimento sobre (e consequentemente compreender melhor) um certo domínio; (ii) Ajudar as pessoas a atingir um consenso conceitual sobre um domínio; e (iii) Ajudar as outras pessoas a compreender um certo domínio sem a necessidade de um especialista [Duarte and Falbo 2000].

\section{Fronteiras da educação}

Nesta seção apresentamos uma visão sobre o funcionamento das práticas de educação, ainda substancialmente moldadas por modos de ensino tradicionais, podem ser transformadas. A leitura crítica dessas práticas tradicionais de educação implica no levantamento de suas estruturas limitantes, i.e., das suas fronteiras.

Fronteiras nada mais são que uma marcação para determinar limites. Identificamos no nosso sistema de ensino tradicional, cinco fronteiras principais: (i) Físicotemporal, (ii) Virtual, que ainda imita a físico-temporal, (iii) Ausência de interdisciplinaridade (iv) Tipo de atividades que desempenhamos e (v) Hierarquia na aprendizagem [Silveira et al. 2018a].

A fronteira Físico-temporal está relacionada à ideia de que só aprendemos dentro do ambiente escolar, durante um período bem determinado, com hora para início e 
VIII Congresso Brasileiro de Informática na Educação (CBIE 2019)

Anais do XXX Simpósio Brasileiro de Informática na Educação (SBIE 2019)

fim. Este ambiente não está preparado para a revolução educacional que já começou há algum tempo [Silveira et al. 2018a]. Aqui incluímos condições subjetivas por parte de professores e administradores que temem mudanças, principalmente as revolucionárias.

As pessoas estão expandindo sua fonte de aprendizagem além da escola, colocando-a em suas casas, bibliotecas, cafés e locais de trabalho, onde quando e como podem decidir o que querem aprender. Esses novos nichos de aprendizagem atuam na atenuação da fronteira físico-temporal [Collins and Halverson 2018]. Para aprender em todos os lugares em todos os momentos, precisamos de dispositivos digitais que sejam móveis para poderem acessar plataformas e recursos de aprendizagem em qualquer lugar e o tempo todo. Ferramentas de hardware e software são o ponto de partida para os estudantes iniciarem e continuarem suas atividades de aprendizagem continuamente. Consequentemente, já não se assume que a aprendizagem do estudante tenha de começar com um professor. [Jackson and Cooper 2014].

Entendemos que a tecnologia tem um papel muito importante na quebra dessa fronteira. Isto nos leva à segunda fronteira. De acordo com [Moraes 2008] existe um problema na forma de apropriação da tecnologia e no modelo pedagógico que vem sendo utilizado. Apesar de incorporar características que os livros não possuem e de tornar a interface mais agradável, o uso arbitrário de tecnologia continua perpetuando o velho ensino, "otimizando o péssimo". Observamos que a maioria das propostas de uso de tecnologias na educação se apoia numa visão instrucionista, reforçando a fragmentação do conhecimento e, consequentemente, a fragmentação da prática pedagógica. O fato de integrar imagens, textos, sons, animações e mesmo a interligação de informações em sequências de hyperlinks, por si só, não nos dá a garantia de boa qualidade pedagógica e de uma nova abordagem educacional [Moraes 2008].

A terceira fronteira está ligada à necessidade de interdisciplinaridade na grade curricular. Neste contexto, a educação tradicional sofre dos seguintes problemas: (i) A separação em disciplinas descontextualiza a aprendizagem. Acreditamos que os conteúdos podem ser explorados de maneira mais livre como, por exemplo, com trabalho orientado a problemas [Boud and Feletti 2013]; (ii) O currículo tradicional, estruturado em torno das grades disciplinares, faz com que o estudante aprenda fragmentadamente. É necessário um novo currículo, dotado de visão holística e integradora, com cuidados essenciais nas diferentes dimensões da aprendizagem, sejam elas individuais, coletivas ou planetárias [Albuquerque et al. 2008]. Assim, concordamos com o conceito "lifewide curriculum" [Jackson and Cooper 2014] que se destina a complementar as formas mais tradicionais de currículo acadêmico, abrangendo todas as experiências de um estudante. As proposições levam em conta a necessidade de os estudantes resolverem as coisas por si mesmos enquanto se envolvem, intencional ou acidentalmente, nas experiências que encontram ou criam no mundo real/virtual.

A quarta fronteira relaciona-se ao tipo de atividade desempenhada, no que torna obrigatório aprender apenas quando estamos estudando. No entanto as pessoas criam nichos (ou ecossistemas) de aprendizagem completamente diferentes para as suas tarefas cotidianas. Esses nichos refletem os diferentes contextos, propósitos e atividades que geram cognições nas pessoas. Eles envolvem atividades com a família e amigos, com os colegas de trabalho, com companheiros em esportes, na busca de hobbies etc. Aí está uma grande oportunidade de construir conhecimento e alcançar objetivos de apren- 
VIII Congresso Brasileiro de Informática na Educação (CBIE 2019)

Anais do XXX Simpósio Brasileiro de Informática na Educação (SBIE 2019)

dizagem. Apesar de contribuírem significativamente para o desenvolvimento, sucesso e bem-estar dos indivíduos [Jackson and Cooper 2014], essas esferas cognitivas raramente são consideradas pelos métodos pedagógicos tradicionais, entretanto, a aprendizagem está embutida no processo de viver [Rogers 2003].

A quinta e última fronteira está relacionada à determinação sobre alguém que aprende e outro que ensina. Em geral, as interações para construção de conhecimento presumem uma hierarquia, ou seja, o professor fala e o estudante escuta. No entanto, temos vivenciado experiências com projetos de aprendizagem (o professor como mediador) que têm demonstrado que isso é uma limitação associada à tradição da escola. O principal problema com relação a essa fronteira é o estudante acreditar ser totalmente dependente do professor para construir seu conhecimento. Segundo [Zhang et al. 2010], os estudantes precisam demonstrar habilidades de planejamento, análise, raciocínio e avaliação, bem como desenvolver habilidades metacognitivas para gerenciar suas próprias jornadas de aprendizagem. Ademais, quando as escolas estão conectadas à Internet, é muito perceptível como o uso de computadores energiza os estudantes e torna a sala de aula um ambiente de aprendizagem mais interativo, onde a aprendizagem pode fluir paralelamente e não apenas na vertical. [Kirkman et al. 2002].

\section{Ontologia para ecossistemas de aprendizagem}

A pesquisa e estudo sobre os ecossistemas de aprendizagem é muito recente e carente de conceituação e definições que sejam compartilhadas pela comunidade científica. Em síntese, o entendimento sobre ecossistemas de aprendizagem considerando a cultura digital apresenta problemas, tais como: (i) complexidade do domínio, envolvendo conceitos e relações e (ii) falta de clareza e precisão semântica na descrição dos conceitos do domínio.

Nesta seção apresentamos, a formalização da Ontologia de Referência para

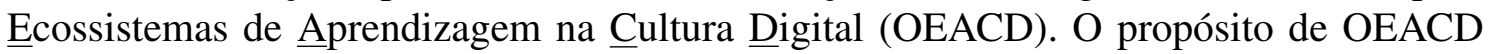
é prover um modelo de referência que forneça um consenso sobre os conceitos e relações em ecossistemas da aprendizagem na cultura digital. OEACD possui dois usos pretendidos: (i) Oferecer suporte a especialização e instanciação de novos EACD; e (ii) Possibilitar a avaliação de propostas de ecossistemas de aprendizagem na cultura digital, ou seja, fornecer elementos de comparação para EACD.

\subsection{Metodologia}

A ontologia foi desenvolvida com base no método SABiO (Systematic Approach for Building Ontologies) [Falbo 2014]. Este é um método de engenharia de ontologia sólido e que provê um conjunto de atividades e diretrizes para a especificação de ontologias com uma abordagem focada na criação de ontologias de domínio. O método é realizado em um processo composto de cinco fases: (i) Identificação do propósito e usos pretendidos, bem como a definição de requisitos (questões de competência); (ii) Captura e formalização; (iii) Projeto de refinamento; (iv) Implementação usando uma linguagem operacional, por exemplo OWL; e (v) Testes de verificação e validação da ontologia [Falbo 2014]. Devido a ausência de espaço neste documento, apresentaremos apenas parte das fases (ii) e (iii) de OEACD. O documento completo de especificação da ontologia pode ser acessado em um link nas referências [Silveira and Oliveira 2018].

Para a formalização da OEACD, utilizamos a OntoUML [Guizzardi 2007], que lança mão tanto meta-propriedades ontológicas quanto categorização de objetos. Isso é 
VIII Congresso Brasileiro de Informática na Educação (CBIE 2019)

Anais do XXX Simpósio Brasileiro de Informática na Educação (SBIE 2019)

feito com a introdução de estereótipos no diagrama de classes para de fato obter uma caracterização ontológica consistente. A Tabela 1 apresenta os estereótipos usados neste artigo. Nela, um tipo $\mathrm{T}$ é rígido se para toda instância $\mathrm{x}$ de $\mathrm{T}$, $\mathrm{x}$ é necessariamente uma instância de $\mathrm{T}$ (no senso modal). Em outras palavras, se $\mathrm{x}$ instancia $\mathrm{T}$ em um dado mundo w, então x deve instanciar T em todo mundo possível w [Detoni et al. 2019]

Tabela 1. Estereótipos OntoUML [Detoni et al. 2019] (estendido)

\begin{tabular}{ll}
\hline \multicolumn{1}{c}{ Estereótipo } & \multicolumn{1}{c}{ Descrição } \\
\hline$<<$ kind $>>$ & Tipos rígidos que fornecem princípio de identidade para suas instâncias. \\
$<<$ subkind $>>$ & $\begin{array}{l}\text { Tipos rígidos cujas instâncias especializam instâncias de um }<<\text { kind }>> \\
\text { Tipos rígidos que capturam propriedades comuns entre entidades que pos- } \\
\text { suem diferentes princípios de identidade. }\end{array}$ \\
$<<$ category $>>$ & Tipos anti-rígidos instanciados no escopo de uma relação. \\
$<<$ role $>>$ & Tipos anti-rígidos que generalizam roles (diferente critério de identidade). \\
$<<$ roleMixin $>>$ & $\begin{array}{l}\text { Tipo que reifica uma relação e cujas instâncias são dependentes existenci- } \\
\text { almente das entidades relacionadas. }\end{array}$ \\
$<<$ relator $>>$ & $\begin{array}{l}\text { Características associados a uma estrutura de qualidade, ou seja, um espaço } \\
\text { conhecido de valores, como por exemplo cores. }\end{array}$ \\
$<<$ quality $>>$ & $\begin{array}{l}\text { Características associados a algo que não pode ser medido com valores, } \\
\text { como por exemplo intenção ou desejo. }\end{array}$ \\
$<<$ mode $>>$ &
\end{tabular}

\subsection{Formalização da ontologia}

OEACD é dividida em três subontologias: (i) Ambiente Educacional - AE (cor cinza na Figura 1): É uma subontologia secundária que possui conceitos compartilhados entre as próximas subontologias. Aborda principalmente a mediação pedagógica e as relações de utilização de ferramentas em ambientes de aprendizagem a partir da execução de um plano de trabalho; (ii) Arquitetura Pedagógica - AP (cor branca na Figura 1): É uma subontologia secundária, que compreende uma estratégia de ensino que pode ser aplicada em uma EACD. As arquiteturas pedagógicas têm componentes informativos e propositivos, e o objetivo é ajudar a autonomização dos estudantes [Carvalho et al. 2005]; (iii) Ecossistemas de Aprendizagem na cultura Digital - EACD (amarelo na Figura 1). É a subontologia principal e compreende os elementos envolvidos no ecossistema de aprendizagem como um todo, o que inclui principalmente os agentes, o ambiente e as interações.

Observando a Figura 1, vemos que todo EACD acontece em um ambiente onde existem agentes interagindo. Esse ambiente pode ser composto por outros subambientes que podem ser reais (por exemplo, a sala de aula) ou virtuais (por exemplo, um grupo de conversação de telefone). Como estamos trabalhando com ecossistemas de aprendizagem considerando a cultura digital, o ambiente deve conter pelo menos um subambiente virtual e até mesmo nenhum ambiente real.

Existem dois tipos de interação: (i) Agente x Agente e (ii) Agente x Ambiente. A Figura 1 mostra que um registro de aprendizagem é inerente a um Agente Aprendiz mas é externamente dependente de um Agente Interagente ou do Ambiente (tipos de interação (i) e (ii) respectivamente). Ainda na Figura 1, observando a subontologia AE, assumimos que um Recurso Pedagógico pode ser Virtual ou Real. O estereótipo $<<$ roleMixin $>>$ nos diz que um objeto (virtual ou real) passa a ser um recurso pedagógico quando atrelado 
VIII Congresso Brasileiro de Informática na Educação (CBIE 2019)

Anais do XXX Simpósio Brasileiro de Informática na Educação (SBIE 2019)

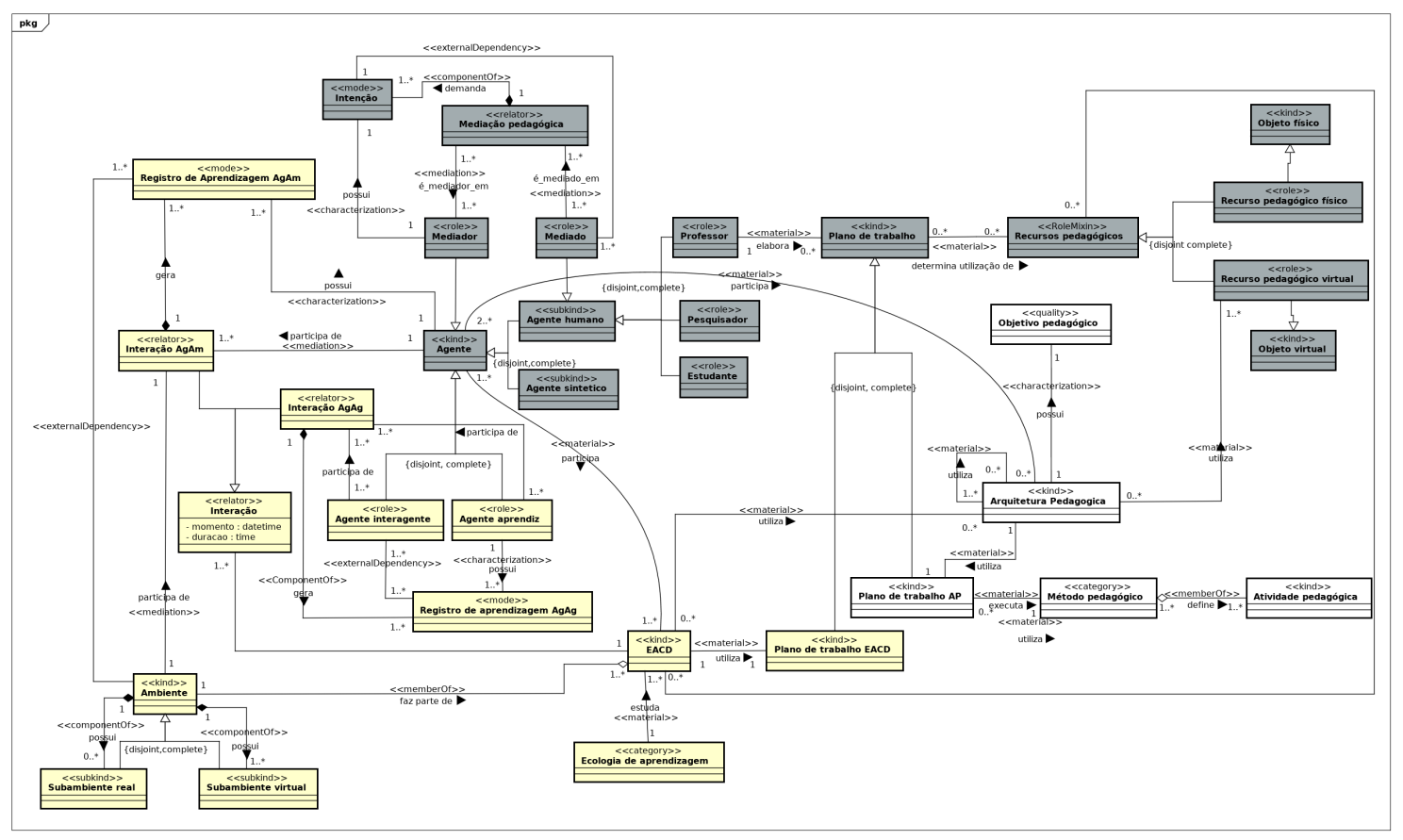

Figura 1. Diagrama OntoUML para OEACD

a um plano de trabalho, ou seja, quando destinado a ser usado como ferramenta para potencializar aprendizagens. Por exemplo, um blog é apenas um blog, mas quando utilizado como um portfólio de aprendizagem passa a ser um recurso pedagógico virtual.

\section{Ecossistemas de aprendizagem como solução para fronteiras da educação}

[Brown 2000] usa "ecologia"em consonância com "ecossistema", como uma metáfora para descrever um cenário de aprendizagem, conceitualizando-o como um sistema adaptativo aberto e complexo que compreende elementos dinâmicos e interdependentes, de forma que uma das coisas que torna um ecossistema tão poderoso e adaptável a novos contextos é a sua diversidade. Isso, em resumo, alimenta um indício de que os ecossistemas de aprendizagem podem fornecer a solução que procuramos.

[Richardson 2002] quando fala sobre "Toward an Ecology of Learning", traz algumas características que determinam como se estabelece um ecossistema de aprendizagem. A seguir discutiremos como elas podem atuar na superação das fronteiras da educação.

\subsection{Fronteiras 1 e 2}

Um ecossistema de aprendizagem bem-sucedido oferece um ambiente de aprendizagem ubíquo com acesso a qualquer momento, e em qualquer lugar. Os alunos têm acesso aberto e imediato ao ecossistema, onde podem procurar, localizar e acessar rapidamente elementos de aprendizagem e para construir e organizar interações personalizadas e exclusivas [Richardson 2002]. Essa é a característica que ajuda na suplantação da fronteira físico-temporal tanto no mundo físico quanto no virtual.

Observando o modelo conceitual da ontologia (Figura 1) um ecossistema deve oferecer suporte para interações síncronas e assíncronas, tanto entre agentes quanto entre agente e ambiente. As interações, fonte dos registros de aprendizagens, são livres e ocorrem independentes de lugar ou tempo determinado. 
VIII Congresso Brasileiro de Informática na Educação (CBIE 2019)

Anais do XXX Simpósio Brasileiro de Informática na Educação (SBIE 2019)

\subsection{Fronteira 3}

O design instrucional e os elementos de conteúdo que formam um ecossistema de aprendizagem são dinâmicos e interdependentes. $\mathrm{O}$ ambiente de aprendizagem deve permitir que elementos concebidos como objetos de conteúdo relevantes sejam reaproveitados dinamicamente em uma variedade de modelos pedagógicos ou em outras palavras aproveitar segmentos de um contexto em outro contexto. Esta reorganização dinâmica do conteúdo em diferentes modelos pedagógicos cria um sistema de aprendizagem que se adapta e favorece relações entre as diferentes atividades dos estudantes [Richardson 2002]. Essa é a característica que ajuda na suplantação da fronteira da ausência de interdisciplinaridade.

Em nossa visão, um mesmo agente pode se encontrar em diferentes EACDs e viceversa (relacionamento N-N entre Agente e EACD no modelo conceitual - Figura 1), o que significa a existência de trocas de contexto e consequente aproveitamento de registros de aprendizagens em diferentes contextos, como por exemplo o aproveitamento de conceitos aprendidos em uma disciplina de engenharia de software (EACD X) em outra disciplina de banco de dados (EACD Y).

\subsection{Fronteira 4}

O conceito de ecologia de [Brown 2000] requer a criação e entrega de um ambiente de aprendizagem que apresente uma diversidade de opções de aprendizagem para o aprendiz oferecendo a ele oportunidades de construir aprendizagem por meio de métodos e modelos que melhor atendam às suas necessidades, interesses e atividades nas quais ele esteja envolvido Essa é a característica que ajuda na suplantação da fronteira relativa às atividades que desempenhamos [Richardson 2002].

Richardson tem o suporte do estudo realizado por [Illeris 1996] que refuta a ideia de que aprendemos apenas quando estamos estudando. Em OEACD isso fica claro quando vemos que os registros de aprendizagem são livres de contexto e podem ser usados por agentes mediadores para ajudar na construção de novas aprendizagens, independente da atividade realizada. Um exemplo disso, é quando se alcança uma aprendizagem no meio acadêmico que pode ser aproveitada no trabalho, ou mesmo em uma atividade doméstica.

\subsection{Fronteira 5}

Um ecossistema de aprendizagem também deve apoiar a aprendizagem social de uma forma geral. O ambiente precisa oferecer recursos tecnológicos para que os alunos formem equipes de aprendizagem em atividades colaborativas ou se auto-organizem em grupos de discussão nos quais possam explorar tópicos de aprendizagem. O ecossistema também deve permitir discussões e compartilhar insights dentro de suas comunidades especializadas de prática, ou seja, em seus EACDs [Richardson 2002]. Aqui suplantamos a fronteira relacionada à hierarquia na aprendizagem.

Observando a ontologia, vemos que os agentes que assumem o papel de aprendizes, podem ser tanto professores, quanto estudantes ou pesquisadores. Além disso, não existe uma relação hierárquica nas interações ou nas mediações, nas quais um mediador sempre será um humano, ou seja, ele pode ser o professor, outro estudante ou até mesmo um agente virtual (IA). Neste contexto, é importante mencionar a subontologia para arquiteturas pedagógicas (APs) disponível em OEACD. Mesmo que não seja obrigatório o uso de APs nos EACDs, o mediador tem em mãos esse recurso poderoso para suplantação 
VIII Congresso Brasileiro de Informática na Educação (CBIE 2019)

Anais do XXX Simpósio Brasileiro de Informática na Educação (SBIE 2019)

dessa fronteira, levando em consideração a atuação das APs na transformação do aluno em protagonista na sua aprendizagem.

\section{Considerações finais}

Neste artigo destacamos algumas limitações da escola atual que nomeamos fronteiras da educação. Apresentamos os ecossistemas de aprendizagem sob a luz do modelo conceitual de OEACD, bem uma discussão sobre como os ecossistemas de aprendizagem podem auxiliar na suplantação das referidas fronteiras da educação.

Os ecossistemas devem ser potencializados por aplicações tecnológicas que auxiliem os estudantes a: (i) evitar tarefas desnecessárias, (ii) ajudar na captura daquilo que seus sentidos podem requerer, (iii) perceber em que devem focar em contextos onde muitas coisas acontecem ao mesmo tempo e (iv) buscar, registrar e recuperar mais informação, daquilo que está sendo aprendido. Como trabalho futuro, desenvolveremos uma concepção tecnológica baseada na OEACD que será aplicada a um curso de aperfeiçoamento com um currículo diferenciado.

\section{Referências}

Albuquerque, V. S., Tanji, S., Gomes, A. P., and Siqueira-Batista, R. (2008). Pressupostos da construçāo de um novo currículo para o curso de enfermagem. Rev. Enferm. UFPE on line, 2(4):462-473.

Boud, D. and Feletti, G. (2013). The challenge of problem-based learning. Routledge.

Brown, J. S. (2000). Growing up digital: How the web changes work, education, and the ways people learn. Change Magazine, pages 11-20.

Carvalho, M. J. S., de Nevado, R. A., and de Menezes, C. S. (2005). Arquiteturas pedagógicas para educação à distância: concepções e suporte telemático. In Brazilian Symposium on Computers in Education (Simpósio Brasileiro de Informática na Educação-SBIE), volume 1, pages 351-360.

Castells, M. (2008). Creatividad, innovación y cultura digital. un mapa de sus interacciones. Telos: Cuadernos de comunicación e innovación, 77:50-52.

Collins, A. and Halverson, R. (2018). Rethinking education in the age of technology: The digital revolution and schooling in America. Teachers College Press.

Detoni, A. A., Fonseca, L. B. R., Almeida, J. P. A., and Falbo, R. A. (2019). Uma ontologia de referência para autorização orçamentária e execução da despesa pública. iSys-Revista Brasileira de Sistemas de Informação, 11(3):4-53.

Duarte, K. C. and Falbo, R. A. (2000). Uma ontologia de qualidade de software. Workshop de Qualidade de Software, pages 275-285.

Echeita, G. and Martin, E. (1995). Interação social e aprendizagem. desenvolvimento psicológico e educação: necessidades educativas especiais e aprendizagem escolar. Porto Alegre: Artes Médicas, 3:36-53.

ECOSSISTEMA (2019). Dicionário online Michaelis. Acesso em 17 abr. 2019.

Falbo, R. (2014). Sabio : Systematic approach for building ontologies an overview of sabio. In 1st Joint Workshop Onto.Com/ODISE on Ontologies in Conceptual Modeling and Information Systems Engineering. 
VIII Congresso Brasileiro de Informática na Educação (CBIE 2019)

Anais do XXX Simpósio Brasileiro de Informática na Educação (SBIE 2019)

Germain, C. B. and Gitterman, A. (1995). Ecological perspective. Encyclopedia of social work, 1:816-824.

Guarino, N. (1998). Formal ontology and information systems. In Proceedings of the International Conference on Formal Ontology and Information Systems (FOIS), pages 3-15.

Guizzardi, G. (2007). On ontology, ontologies, conceptualizations, modeling languages, and (meta) models. Frontiers in artificial intelligence and applications, 155:18.

Illeris, K. (1996). Læring, udvikling og uddannelse. Nordisk Pedagogik, 1.

Jackson, N. and Cooper, B. (2014). Life-wide learning, education and personal development e-book. Lifewide Education. Accessed, Guildford.

Kirkman, G., Cornelius, P., Sachs, J., and Schwab, K. (2002). The global information technology report 2001-2002. Oxford, New York.

Moraes, M. C. (2008). O paradigma educacional emergente: implicações na formação do professor e nas práticas pedagógicas. Em aberto, 16(70).

Perret-Clermont, A. N. (1980). Social interaction and cognitive development in children. Academic Press.

Piaget, J. (1973). As operações lógicas e a vida social. Estudos sociológicos, pages 164-193.

Piaget, J., Val, D., and Antonio, J. (1970). La epistemología genética. A. Redondo.

Richardson, A. (2002). An ecology of learning and the role of elearning in the learning environment. Global Summit of Online Knowledge Networks, pages 47-51.

Rogers, A. (2003). What is the difference? a new critique of adult learning and teaching. NIACE, Leicester.

Silveira, P. D. N. and Oliveira, J. (2018). Documento de Especificação de Ontologia de Referência para Ecossistemas da Aprendizagem na Cultura Digital. Disponível em $<$ http://200.137.66.25/TR/OEACD.pdf $>$.

Silveira, P. N., Cury, D., and de Menezes, C. (2018a). Um framework para ecologia da aprendizagem na cultura digital. In Brazilian Symposium on Computers in Education (Simpósio Brasileiro de Informática na Educação-SBIE), volume 29, page 198.

Silveira, P. N., de Menezes, C., Cury, D., and Brito, J. O. (2018b). Uma experiência de mediação em ecossistemas de aprendizagem. In Anais dos Workshops do Congresso Brasileiro de Informática na Educação, volume 7, page 886.

Smart, P., Heersmink, R., and Clowes, R. W. (2017). The cognitive ecology of the internet. In Cognition beyond the brain, pages 251-282. Springer.

Zhang, B., Looi, C. K., Seow, P., Chia, G., Wong, L. H., Chen, W., So, H., Soloway, E., and Norris, C. (2010). Deconstructing and reconstructing: Transforming primary science learning via a mobilized curriculum. Computers and Education, 55(4):15041523. 